\title{
Intervenção educacional no conhecimento dos enfermeiros relacionado ao exame
}

\section{físico da criança}

\author{
Educational intervention in nurses' knowledge related to the child's physical examination \\ Intervención educativa en el conocimiento de las enfermeras relacionado con el examen físico del
}

niño

Recebido: 06/04/2021 | Revisado: 15/04/2021 | Aceito: 20/04/2021 | Publicado: 05/05/2021

Ieda Beatriz dos Santos Peixoto

ORCID: https://orcid.org/0000-0002-6667-6121

Universidade Federal de Pernambuco, Brasil E-mail: ieda.beatriz@ufpe.br

Jéssica Andrade da Fonseca

ORCID: https://orcid.org/0000-0003-0678-630X

Universidade Federal de Pernambuco, Brasil

E-mail: jessicaandrade_20@hotmail.com

Maria Benegelania Pinto

ORCID: https://orcid.org/0000-0002-4333-5439

Universidade Federal de Pernambuco, Brasil

E-mail: maria.benegelania@ufpe.br

Eliane Rolim de Holanda

ORCID: https://orcid.org/0000-0001-6433-9271

Universidade Federal de Pernambuco, Brasil

E-mail: eliane.rolim@ufpe.br

Adriana Montenegro de Albuquerque

ORCID: https://orcid.org/0000-0002-2589-0324

Universidade Federal de Campina Grande, Brasil

E-mail: montenegroadrianaa@gmail.com

Maria Amélia de Souza

ORCID: https://orcid.org/0000-0002-2626-7657

Universidade Federal de Pernambuco, Brasil

E-mail: amelia.souza@ufpe.br

\begin{abstract}
Resumo
Objetivou-se avaliar a intervenção educativa no conhecimento dos enfermeiros relacionado ao exame físico da criança realizada no Município de Vitória de Santo Antão, Pernambuco. Pesquisa quantitativa, quase experimental, exploratório-descritiva. Os dados foram registrados no programa de informática SPSS versão 20.0, e analisados por meio de estatística descritiva e inferencial uni e bivariada, no qual foi aprovada pelo CAAE $\mathrm{n}^{\circ}$ 29766120.9.0000.9430. Participaram da intervenção 27 enfermeiros, a maioria do sexo feminino (96,3\%), com idade média de 34,85 anos e com tempo médio de atuação na Estratégia de Saúde da Família de 4,26 anos. Após a intervenção, os participantes acertaram uma média de 20,54 questões, caracterizando diferenças estatisticamente significativas entre as situações. Evidencia-se que a intervenção educativa proporcionou o aumento do conhecimento dos enfermeiros sobre exame físico da criança na atenção primária à saúde, e enfatiza-se que a capacitação proporcionará mudanças no processo de trabalho. Desse modo, a realização de atividades de intervenção educativa faz-se imprescindível para a prática assistencial do enfermeiro.
\end{abstract}

Palavras-chave: Crianças; Exame físico; Intervenção educativa; Conhecimento; Enfermagem.

\begin{abstract}
The objective of this study was to evaluate the educational intervention in nurses' knowledge related to the physical examination of the child performed in the city of Vitória de Santo Antão, Pernambuco. Quantitative, quasiexperimental, exploratory-descriptive research. The data were recorded in the SPSS software version 20.0, and analyzed using univariate and bivariate descriptive and inferential statistics, which was approved by CAAE $\mathrm{n}^{\circ}$ 29766120.9.0000.9430. Twenty-seven nurses participated in the intervention, most of them female (96.3\%), with an average age of 34.85 years and with an average time in the Family Health Strategy of 4.26 years. After the intervention, the participants answered an average of 20.54 questions correctly, characterizing statistically significant differences between the situations. It is evident that the educational intervention provided an increase in nurses' knowledge about physical examination of children in primary health care, and it is emphasized that training will
\end{abstract}


provide changes in the work process. Thus, the performance of educational intervention activities is essential for the nurse's care practice.

Keywords: Kid; Physical exam; Educational intervention; Knowledge; Nursing.

\section{Resumen}

El objetivo de este estudio fue evaluar la intervención educativa en el conocimiento de las enfermeras relacionada con el examen físico del niño realizada en la ciudad de Vitória de Santo Antão, Pernambuco. Investigación cuantitativa, cuasi-experimental, exploratorio-descriptiva. Los datos se registraron en el software SPSS versión 20.0 y se analizaron mediante estadística descriptiva e inferencial univariante y bivariada, el cual fue aprobado por CAAE $\mathrm{n}^{\circ}$ 29766120.9.0000.9430. Participaron de la intervención 27 enfermeras, la mayoría mujeres (96,3\%), con una edad media de 34,85 años y un tiempo medio en la Estrategia Salud de la Familia de 4,26 años. Tras la intervención, los participantes respondieron correctamente una media de 20,54 preguntas, caracterizando diferencias estadísticamente significativas entre las situaciones. Es evidente que la intervención educativa brindó un incremento en el conocimiento de las enfermeras sobre el examen físico de los niños en la atención primaria de salud, y se enfatiza que la capacitación generará cambios en el proceso de trabajo. Por tanto, la realización de actividades de intervención educativa es fundamental para la práctica asistencial del enfermero.

Palabras clave: Niño; Examen físico; Intervención educativa; Conocimiento; Enfermería.

\section{Introdução}

O acompanhamento dos dois primeiros anos de vida da criança, faz-se de extrema importância, visto que é nesta faixa etária que ocorrem as variadas aquisições de habilidades no decorrer do processo de desenvolvimento, além do acelerado crescimento, considerado, portanto, como um período crítico, passível a vulnerabilidades provenientes de agravos socioeconômicos e ambientais (Nascimento, Lemos, Alves, Silva, Santos, Vieira \& Souza, 2020).

Para tal, a puericultura caracteriza-se como consulta fundamental presente na atenção primária à saúde, através dela, o enfermeiro, passa a atender as reais necessidades apresentadas pela criança e sua família (Goés et al., 2018).

Dentre as atividades exercidas na puericultura, o exame físico da criança é realizado por meio de consultas criteriosas de abordagem integral e cuidadosa, promovendo a identificação de alterações presentes de forma precoce (Aires et al., 2015). O mesmo, deve ser realizado de forma regular e conforme o preconizado pela unidade de saúde (Brito et al., 2015). Ressalta-se que a consulta de enfermagem se trata de uma atividade privativa do enfermeiro, estando disposta na lei do exercício profissional de enfermagem, no 7.498, de 25 de junho de 1986 (Brasil, 1986).

Ao realizar o Exame Físico, o enfermeiro necessita estar embasado cientificamente em variados campos da saúde, devendo o acompanhamento ser realizado de forma sistematizada considerando não apenas os dados subjetivos informados, mas também, a clínica apresentada, além da linguagem não verbal (Santos et al., 2011). Uma vez adotado método científico e sistematizado na prática assistencial de enfermagem, é possível a prevenção e promoção da saúde como também a realização de atividades de proteção e recuperação, qualificando a assistência e reduzindo gastos (Nogueira, 2020; Oliveira, 2013).

Entretanto, enfatiza-se que ainda existem fragilidades no âmbito prático e teórico assistencial prestado pelos enfermeiros da atenção primária à saúde, e para superá-las há a necessidade da realização de atividades como capacitação dos profissionais, possibilitando através disto a formulação de respostas satisfatórias mediante as demandas da atenção primária à saúde (Reicher et al., 2015).

O objetivo dessa pesquisa foi avaliar a intervenção educativa no conhecimento dos enfermeiros relacionado ao exame físico da criança realizada no Município de Vitória de Santo Antão, Pernambuco.

\section{Metodologia}

Estudo quase experimental, do tipo grupo único, antes e depois, não randomizado, com caráter exploratóriodescritivo, de abordagem quantitativa. A pesquisa quantitativa possibilita coletar, analisar os dados quantitativos das variáveis presentes em estudos, no qual possibilita a partir disto, a identificação de realidades, das relações envolvidas, dinâmicas 
estruturais, força de associação ou correlação, assim como, proporcionar a partir das informações coletadas a inferência causal dos fatos que ocorrem ou não. Portanto, a eficiência e eficácia no âmbito da enfermagem são comprovados a partir de abordagens fundamentadas em evidências (Esperón, 2017).

Quanto ao desenho descritivo exploratório utiliza-se o conhecimento sobre determinados acontecimentos ou fenômenos, no qual em particular são escassos. Sendo necessária por parte do pesquisador a observação, descrição, assim como, a documentação dos fatores envolvidos ao fato ocorrido, sendo dispensável a manipulação de variáveis como também a investigação acerca de causa ou efeito, objetivando, portanto, a descrição, frequência e categorização da realidade presente (Benanchio et al., 2017).

O estudo quase experimental é caracterizado por intervenção sem a necessidade de randomização, ou até mesmo a presença de um grupo controle em determinados casos (Dutra et al., 2016).

Intervenção educativa realizada em parceria com a Universidade Federal de Pernambuco - Centro Acadêmico de Vitória (CAV), e secretária de Saúde do Município, a qual disponibilizou o espaço e transporte. Evidencia-se carga horária de quatro horas para a intervenção.

A população foi constituída por todos os enfermeiros $(n=27)$ que integram as equipes da Estratégia de Saúde da Família (ESF), sendo o município da Vitória de Santo Antão constituído por 27 Unidades Básicas de Saúde (UBS), caracterizado como pesquisa censitária. Foram considerados como critérios de elegibilidade: ser enfermeiro adscrito a alguma unidade básica de saúde do município de Vitória de Santo Antão e estar no exercício da profissão no período da coleta de dados. Profissionais que estavam afastados do exercício de sua profissão por motivo de saúde, licença ou férias e participantes que não estiveram presentes em todas as etapas não foram inclusos na pesquisa.

A capacitação apresentou caráter teórico prático, sendo a parte teórica composta por conteúdo programático partindo das considerações gerais sobre o exame físico da criança, seguido pelos métodos antropométricos, onde foram abordadas técnicas de aferição de peso, estatura, índice de massa corpórea (IMC), circunferência abdominal segundo as orientações para a coleta e análise de dados antropométricos em serviços de saúde do Ministério da Saúde (Brasil, 2019). Além do perímetro cefálico e torácico (Brêtas et al., 2012).

Disponibilizou-se para os participantes durante a intervenção um roteiro teórico ministrado, para melhor acompanhamento, assim como, para anotações pertinentes do conteúdo abordado. Salienta-se que a parte prática constou de demonstrações, manuseios com os equipamentos de mensuração antropométricos e realização de manobras para exame físico da criança, objeto desta pesquisa.

O conhecimento prévio dos enfermeiros sobre exame físico da criança foi avaliado por meio da aplicação de um préteste, questionário semiestruturado, construído para o estudo, composto por 13 perguntas subjetivas e 26 objetivas. O instrumento foi validado por um grupo de expertises na área de saúde da criança a fim de observar se o instrumento contemplava as questões inerentes ao estudo. Na sequência, o instrumento foi pré-testado por 3 enfermeiros de modo a garantir sua adequação para a aplicação definitiva. O questionário constituía-se de duas partes, sendo a primeira, de análise sociodemográfica dos participantes seguido de um instrumento de coleta da capacitação em exame físico da criança, com questões específicas, voltadas a realização do exame físico em consonância com as orientações em serviços de saúde da Atenção Primária à Saúde (Brasil, 2019). Considera-se ser o questionário, autoaplicável, no qual foi respondido antes e imediatamente após a capacitação, ou seja, pré e pós-teste, respectivamente.

As variáveis do instrumento abrangiam características sociais e profissionais dos participantes, em destaque o sexo, idade, tempo de conclusão na graduação em enfermagem, titulações, tempo de atuação como enfermeiro na Estratégia de Saúde da Família, tempo de atuação como enfermeiro na Estratégia de Saúde da Família no vínculo atual, participação em capacitações sobre puericultura, participação em Capacitação/Treinamentos/Cursos sobre exame físico da criança, conteúdos 
de interesse dos enfermeiros na realização de curso de capacitação sobre exame físico da criança. Assim como, o quantitativo de atendimentos semanais realizados pelo Enfermeiro que contemplem o exame físico da criança e a presença de dificuldades para realização do exame físico da criança pelo enfermeiro.

Os dados foram registrados no banco de dados do programa de informática SPSS (Statistical Package for Social Sciences) for Windows, versão 20.0, e analisados por meio de estatística descritiva e inferencial uni e bivariada. Para os procedimentos descritivos, foram apresentados os dados brutos e relativos (frequências e porcentagens) e medidas de tendência central (média e mediana) e dispersão (desvio-padrão e valores mínimos e máximos). Os procedimentos de inferência estatística, por sua vez, foram feitos com base em estatística não-paramétrica, em função do quantitativo amostral (n=27), por meio do Teste de Wilcoxon, para avaliação de diferenças entre condições em medidas repetidas. Ressalta-se que, para interpretação das informações, adotou-se um nível de confiança de 95\% e significância de 5\%.

A pesquisa atendeu a Resolução 466/2012 do Conselho Nacional de Saúde que envolve seres humanos e foi aprovada pelo comitê de ética em pesquisa sob o CAAE no 29766120.9.0000.9430. O Termo de Consentimento Livre e Esclarecido (TCLE) foi assinado em duas vias e entregue aos participantes antes da realização da coleta de dados, no pré-teste e no local da realização da intervenção educativa.

\section{Resultados}

Participaram da pesquisa 27 enfermeiros ( $\mathrm{n}=27$ ), a maioria do sexo feminino 96,3\% (26), com idade média de 34,85 anos ( $\mathrm{DP}=8,05)$, variando de 23 a 52 anos. Identifica-se tempo médio de conclusão da graduação de 6,65 anos $(\mathrm{DP}=5,38)$, existindo profissionais entre um e 29 anos de formados. Em relação à titulação, 77,8\% possuíam especialização, não sendo registrados, nessa amostra, casos de mestres e doutores. Quanto à atuação como enfermeiro na Estratégia de Saúde da Família, observa-se tempo médio de 4,26 anos ( $\mathrm{DP}=4,29$; Mín = 0; Máx = 19), e para o vínculo atual, identificou-se o tempo médio de 2,42 anos (DP = 1,86; Mín = 0; Máx = 10).

No que concerne à avaliação da capacitação dos enfermeiros na área de exame físico da criança, questionou-se aos enfermeiros se participaram de alguma capacitação sobre puericultura, no qual 74,1\% (20) confirmaram que sim, 22,2\% (6) afirmaram que não e 3,7\% (1) informaram não lembrar. Na sequência, foram questionados sobre a participação em treinamento/curso/capacitação sobre exame físico da criança, identifica-se que 48,1\% (13) já ter participado, 37,0\% (10) não participaram e 14,8\% (4) declararam não lembrar.

Em relação aos conteúdos de interesse pelos enfermeiros na participação de curso de capacitação sobre exame físico da criança, a não especificação foi correspondente a 33,3\% (9) e a ausculta cardíaca 22,2\% (6). Ressalta-se que as demais respostas estão detalhadas na Tabela 1. 
Tabela 1. Conteúdos de interesse dos enfermeiros na realização de curso de capacitação sobre exame físico da criança.

\begin{tabular}{lcr}
\hline \multicolumn{1}{c}{ Conteúdos de interesse } & $\mathrm{f}$ & $\%$ \\
\hline Alterações do exame físico da criança & 2 & 7,4 \\
Anamnese e Inspeção & 1 & 3,7 \\
Ausculta Cardíaca & 6 & 22,2 \\
Ausculta Pulmonar & 1 & 3,7 \\
Dermatologia & 3 & 11,1 \\
Não especificado & 9 & 33,3 \\
Quaisquer temáticas propostas & 2 & 7,4 \\
Reanimação & 1 & 3,7 \\
Reflexos & 1 & 3,7 \\
Visita puerperal & 1 & 3,7 \\
\hline
\end{tabular}

Fonte: Autores.

Na sequência, avaliou-se o número médio de atendimentos semanais realizados com a execução do exame físico da criança pelos profissionais. Em média são realizados 17,41 atendimentos $(\mathrm{DP}=9,63)$, existindo entre 8 e 40 atendimentos. Foram avaliadas, também, as dificuldades dos profissionais na realização do exame físico nas crianças acompanhadas na ESF e $29,6 \%$ dos profissionais relataram encontrar dificuldades, dentre as quais foram às relacionadas à dermatologia (3,7\%), falta de material adequado $(3,7 \%)$, pouco tempo de atendimento para o elevado quantitativo de crianças $(14,8 \%)$ e resistência da criança ao exame $(7,4 \%)$. Destaca-se que 70,4\% (p< 0,001) dos profissionais não especificaram as dificuldades.

Foi calculada a quantidade de acertos aos questionários nas situações pré e pós-teste. Antes da intervenção, os enfermeiros acertaram, em média, 13,54 questões ( $\mathrm{DP}=3,16$; Mediana $=14,00)$, variando de 6 a 18 questões. Após a intervenção, acertaram uma média de 20,54 questões (DP = 3,33; Mediana = 21,00), oscilando de 14 a 26 questões. Esses dados foram submetidos ao teste inferencial de Wilcoxon, e foram identificadas diferenças estatisticamente significativas entre as situações (Tabela 2).

Tabela 2. Avaliação da quantidade de acertos sobre exame físico da criança em grupos de pré e pós intervenção.

\begin{tabular}{ccccccc}
\hline Grupos & $\mathrm{M} \pm \mathrm{DP}$ & $\mathrm{IC}$ 95\% & Mediana & Mínimo & Máximo & $\mathrm{Z} ; \mathrm{p}$ \\
\hline Pré-intervenção & $13,54 \pm 3,16$ & $12,26-14,82$ & 14,00 & 6 & 18 & $\mathrm{Z}=4,47$ \\
Pós-intervenção & $20,54 \pm 3,33$ & $19,19-21,89$ & 21,00 & 14 & 26 & $\mathrm{p}<0,001$ \\
& & & & & & \\
\hline
\end{tabular}

Fonte: Autores.

\section{Discussão}

Identificou-se no estudo a predominância do público feminino, fato este, advém de um contexto histórico na enfermagem. De acordo com Borges et al., (2017), há a feminização da enfermagem, característica atrelada ao profissional em virtude dos relatos históricos da mulher ao ato de cuidar. Paralelamente, Cunha et al., (2017) enfatiza que apesar da predominância de mulheres na prática profissional da enfermagem, a presença de homens torna-se uma realidade nos últimos anos, ocasionando, portanto, uma ruptura nos estereótipos de gênero associados à profissão do enfermeiro.

O tempo de formação de enfermeiros segundo estudos realizados nos estados de Minas Gerais e Rio Grande do Sul acerca do perfil dos profissionais da atenção primária à saúde, em ambos os estados, apresentaram um quantitativo inferior a dez anos de formação, assim como, o vínculo de trabalho na unidade de saúde apresentava-se recente, com média de 4,5 anos, 
fato semelhante aos resultados obtidos neste estudo para o vínculo de atuação profissional (Santos, 2019; Sturmer, 2020).

Dentre as atividades exercidas pelo enfermeiro na atenção primária à saúde, a puericultura permite a realização do acompanhamento dos marcos do desenvolvimento, atualização da situação vacinal, atividades de educação em saúde, além de outras ações, que impactam diretamente na redução da morbimortalidade infantil (Santos, 2012; Sobral, 2018).

O exame físico inserido no cenário da puericultura, constitui como componente fundamental da atenção à saúde da criança, devendo ser realizado por meio de consultas criteriosas de abordagem integral e cuidadosa, promovendo a identificação de alterações presentes de forma precoce (Aires et al., 2015).

Enfatiza-se, ainda, que é dever do enfermeiro o desenvolvimento das habilidades e conhecimentos necessários para a prática assistencial da puericultura, com enfoque na promoção de ações educativas e participação em práticas de aperfeiçoamento profissional em benefício da criança, família e coletividade (Trindade et al., 2019).

A sobrecarga de trabalho do profissional de enfermagem é uma realidade comum no Brasil, fato concluído por Sousa et al., (2020) em seu estudo acerca das limitações da sistematização da enfermagem. Achado também observado no presente estudo no qual foram explicitados por parte de alguns participantes o pouco tempo de atendimento para o elevado quantitativo de crianças.

Conforme Suto et al., (2014), as dificuldades apresentadas pelo enfermeiro transcendem, há falta de consultórios de enfermagem com estrutura adequada para o atendimento do enfermeiro a criança, sendo comum a realização de adaptações de unidades de saúde, com salas desprovidas de conforto e privacidade para entrevista com os genitores. $\mathrm{O}$ autor enfatiza a ausência de materiais adequados para realização de consultas, muitas vezes, apresentando-se em quantitativo insuficiente e, consequentemente, interferindo na qualidade da assistência prestada pelos enfermeiros.

Um ponto que merece atenção é a resistência da criança ao exame físico, que pode se intensificar em virtude da falta de sensibilização e conhecimento técnico-científico dos profissionais ao atendimento infantil. Ambientes desconhecidos, fora do contexto da criança, podem gerar medos e anseios, principalmente tratando-se de traumas pregressos provenientes do internamento hospitalar (Silva et al., 2020). O ato de brincar, portanto, advém como ferramenta imprescindível a atenção humanizada à saúde da criança (Moraes et al., 2016).

Portanto, faz-se necessário a elaboração de estratégias que reduzam os anseios e medos gerados pela experiência da criança em decorrência dos procedimentos clínicos realizados para prevenção ou manutenção da saúde. Em meio a este cenário, surge o brinquedo terapêutico (Bordoni et al., 2019).

Pontes et al., (2015), em estudo acerca da utilização do uso do brinquedo terapêutico quanto ao comportamento da criança para a realização da vacinação apresentou como resultado a colaboração e quietude dos participantes. A técnica consiste em preparar a criança para as intervenções que serão realizadas pelos profissionais, entretanto segundo Veiga et al., (2016) o uso do brinquedo não exclui as dificuldades apresentadas no âmbito de trabalho da enfermagem.

Com relação à participação em atividades de capacitação profissional acerca da puericultura, houve uma predominância da amostra em participações anteriores, ao tratar-se especificamente sobre o exame físico da criança, os resultados reduziram a menos da metade da totalidade. Alerta-se, portanto, quanto à necessidade de promoção de atividades educativas, como capacitações, com temáticas específicas e de abordagem aprofundada acerca da puericultura.

No que concerne à realização da intervenção educacional com os enfermeiros identificou-se diferenças estatisticamente significativas, com acerto médio de 20,54 questões, caracterizando o aumento do conhecimento dos profissionais a partir da realização de atividades de capacitação. Em estudo semelhante, voltado a atenção a saúde da criança, realizado por Nascimento et al., (2020), com Agentes Comunitários de Saúde (ACS), quanto a realização de antropometria da criança na atenção primária, comprovou-se o aumento do conhecimento acerca da antropometria através de realização de intervenções educacionais, a partir da promoção de capacitações profissional. 
Segundo Oliveira et al., (2016), após análise, a partir de estudo transversal, da formação e qualificação dos profissionais da atenção básica, concluiu que a realização de atividades de capacitação profissional está diretamente relacionada ao aprimoramento da qualidade da prática assistencial. Vieira et al., (2018) por sua vez, complementa, enfatizando que tratando-se do cenário da puericultura, a implementação de intervenções educacionais, como a capacitação dos enfermeiros, possibilita não apenas a prevenção, mas também, a promoção da saúde da população infantil a partir do uso de tecnologias leves.

Entretanto, o autor ressalta que ainda há uma escassez de estudos que elucidem os métodos de ensino e aprendizagem e as estratégias adotadas para realização das capacitações, visando a implementação na Política de Educação Permanente (Oliveira et al, 2016). Para Ferreira et al., (2019), a educação permanente é facilitadora do processo de aprendizagem, tomando como base a promoção de processos de capacitação e formação profissional para suprir as necessidades profissionais.

Silva et al., (2019) enfatiza que os programas de educação permanente, capacitação e formação profissional foram criados a fim de solucionar dificuldades apresentadas na prática profissional, apresentando como benefício o fortalecimento do Sistema Único de Saúde (SUS), como também, a promoção do trabalho em conjunto das instituições de ensino e os componentes do sistema de saúde, de forma a proporcionar uma atenção integral e de qualidade aos usuários.

A realização de intervenções educativas voltadas para a capacitação dos profissionais da atenção primária também é observada em outros estudos acerca de diferentes temáticas vivenciadas no cotidiano da unidade básica de saúde, para Meira Júnior et al., (2016) a realização de capacitações dos profissionais apresenta impacto positivo, sendo complementado por Peixoto et al., (2015), no qual afirma que a realização de intervenções educativas promove o desenvolvimento integral do profissional, agregando conhecimentos e gerando autonomia.

Entretanto, pesquisadores sugerem a incrementação de atividades que reforcem a qualidade dessas intervenções, como a necessidade de reavaliações acerca da continuidade dos conhecimentos e habilidades adquiridas, um bom planejamento das capacitações profissionais além da necessidade da realização periódica da intervenção educativa, sendo papel da educação permanente a articulação e criação de estratégias para a atualização dos profissionais (Manhães, 2015; Meira júnior, 2016).

Souza et al., (2018) sugere como estratégia para realização de intervenções educacionais aos profissionais da saúde, a parceria entre as unidades básicas com as instituições de ensino, integrando o ensino e a pesquisa com a finalidade de criar estratégias que aprimorarem a capacitação dos profissionais.

\section{Conclusão}

É possível concluir que a intervenção educativa proporcionou o aumento significativo do conhecimento dos participantes sobre exame físico da criança na atenção primária. A realização de atividades de atualização profissional, está diretamente relacionado a assistência fornecida pelos enfermeiros, visto que proporcionará a aplicabilidade dos conhecimentos adquiridos no âmbito de trabalho. A promoção de estratégias educativas permanentes, faz-se, portanto, imprescindível para a prática assistencial do profissional.

Este estudo apresentou como limitação a ausência da reavaliação, a longo prazo, do conhecimento dos enfermeiros acerca da atividade educativa realizada, não sendo possível identificar a permanência do aprendizado adquirido pelos participantes.

Ressalta-se que os profissionais almejam participar de mais atividades educativas relacionadas o exame físico da criança, com adoção de temáticas específicas dentro do campo do exame físico, colaborando para realização de estudos posteriormente.

Deste modo, sugere-se que, em pesquisas futuras, se amplie o olhar a respeito dessa temática e que a mesma possa ser aprofundada, valorizando variáveis que são importantes e que até então não tenha tanta visibilidade no contexto da atenção 
primária à saúde, além de mobilizar enfermeiros/pesquisadores para a realização de outros estudos a fim de buscar outras evidências que não foram investigadas nesta pesquisa.

Por fim, enfatiza-se que há uma escassez na literatura de artigos que abordem sobre o exame físico da criança, esperase, portanto, que os resultados apresentados neste estudo possam demonstrar a relevância de atividades de intervenção educativa e da realização do exame físico da criança aos profissionais e estudantes de graduação em saúde além de contribuir para posteriores estudos no âmbito da saúde.

\section{Referências}

Aires, L. C. P., Santos, E. K. A., Costa, R., Borck, M., \& Custódio, Z. A. O. (2015). Seguimento do bebê na atenção básica: interface com a terceira etapa do método canguru. Revista Gaúcha de Enfermagem, 36(esp), 224-32.

Benachio, E. C. C., Mendonça, P. H., \& Nascimento, F. L. S. (2019). Procedimentos metodológicos empregados nos artigos public ados na revista brasileira da educação profissional e tecnológica. Revista Temas e Educação, 28(1), 60-75.

Bordoni, C. J., Irmgard, B. G., R., Damé, H. T., Gonçalves, V. V. S. M. R., \& Marten, M. V. (2019). O brinquedo terapêutico no cuidado à criança hospitalizada. Revista Enfermagem Atual In Derme, 88(26).

Borges, T. M. B., \& Detoni, P. P. (2017). Trajetórias de feminização no trabalho hospitalar. Cadernos de Psicologia Social do Trabalho, $20(2), 143-157$.

Brasil. (2019). Caderneta de saúde da criança: Crescimento e desenvolvimento. Passaporte da cidadania 12 edição. Ministério da Saúde. Brasília. Governo Federal.

Brasil. (1986). Lei 7.498, de 25 de junho de 1986. Dispõe sobre a Regulamentação do Exercício da Enfermagem e dá outras providências. Brasília: Ministério da Saúde.

Brêtas, J. R. S., Quirino, M. D., Silva, C. V., Sabatés, A. L., Ribeira, C. A., Borba, R. I. H., \& Almeida, F. A. (2012). Manual do exame físico para a prática da enfermagem em pediatria. Látria.

Brito, G. V., Albuquerque, I. M. N., Ribeirão, M. A., Moreira, R. M. M., \& Linhares, M. M. C. (2018). Consulta de puericultura na estratégia saúde da família: Percepção de enfermeiros. Revista de Atenção Primária a Saúde, 21(1), 48-55.

Cunha, Y. F., \& Sousa, R. R. (2017). Gênero e Enfermagem: um ensaio sobre a inserção do homem no exercício da enfermagem. Revista de administração Hospitalar e iniciação em saúde, 13(3), 140-149.

Dutra, H. S., \& Reis, V. N. (2016). Desenhos de estudos experimentais e quase-experimentais: definições e desafios na pesquisa em enfermagem. Revista de Enfermagem UFPE, 10(6), 2230-2241

Esperón, J. M. T. (2017). Pesquisa Quantitativa na Ciência da Enfermagem. Escola Anna Nery, 21(1).

Ferreira, L., Barbosa, J. S. A., Esposti, C. D. D., \& Cruz, M. M. (2019). Educação Permanente em Saúde na atenção primária: uma revisão integrativa da literatura. Revista Saúde debate, 43(120). 223-239.

Góes, F. G. B., Silva, M. A., Paula, G, K., Oliveira, L. P. M., Mello, N. C., \& Silveira, S. S. D. (2018). Contribuições do enfermeiro para boas práticas na puericultura: revisão integrativa da literatura. Revista Brasileira Enfermagem, 71(6), 2974-2983.

Manhães, L, S, P., Pinto, A. C. S., Izu, M., Tavares, C. M. M., \& Rosas, A. M. M. F. T. (2015). Percepção de enfermeiros em relação ao treinamento em serviço oferecido pelo serviço de educação permanente. Revista de Pesquisa: cuidado é fundamental, 7(2), $2323-2335$.

Moraes, A. C. C., \& Lima, C. A. (2016). Brinquedoteca: a importância do brincar para a criança hospitalizada. Revista GeoPantanal, $11($ esp), $131-145$.

Meira Júnior, L. E., Souza, F. M., Almeida, L. C., Veloso, G. G. V., \& Caldeira, A. P. (2016). Avaliação de treinamento em suporte básico de vida para médicos e enfermeiros da atenção primária. Revista Brasileira de Medicina de Família e Comunidade, 11(38), 1-10.

Nascimento, T. R. C., Lemos, G. T. L., Alves, S. M. L., Silva, S. B., Santos, J. A. B., Vieira, C. M., \& Souza, M. A. (2020). Avaliação do conhecimento e intervenção educativa com agentes comunitários de saúde sobre antropometria. Brazilian Journal of Health Review, 3(4), 8032-8048.

Nogueira, D. M. C., Rouberte, E. S. C., Leal, F. K. F., Chaves, C. S., Moura, A. D. A., \& Pinto, L. M. B. (2020). Consultas de puericultura: Avaliação de instrumento para sistematização da assistência de enfermagem. Brazilian Journal of development, 6(5), 32619-32631.

Oliveira, M. P. R., Menezes, I. H. C. F., Sousa, L. M., \& Peixoto, M. R. G. (2016). Formação e Qualificação de Profissionais de Saúde: Fatores Associados à Qualidade da Atenção Primária. Revista Brasileira de Educação Médica, 40(4), 547-559.

Oliveira, F. F. S., Oliveira, A. S. S., Lima, L. H. O., Marques. M. B., Felipe, G. F., \& Sena, I. V. O. (2013). Consulta de puericultura realizada pelo enfermeiro na estratégia saúde da família. Revista rede de Enfermagem do nordeste, 14(4), 694-703.

Pontes, J. E. D., Tabet, E., Folkmann, M. A. D., Cunha, M. L. R., \& Almeida, F. A. (2015). Brinquedo terapêutico: preparando a criança para a vacina. Eistein, 13(2), 238-242. 
Research, Society and Development, v. 10, n. 5, e24110514734, 2021

(CC BY 4.0) | ISSN 2525-3409 | DOI: http://dx.doi.org/10.33448/rsd-v10i5.14734

Reichert, A. P. S., Nóbrega, V. M., Damasceno, S. S., Collet, N., Eickmann, S. H., \& Lima, M. C. (2015). Vigilância do desenvolvimento infantil: práticas de enfermeiras após capacitação. Revista Eletrônica de Enfermagem, 17(1), 117-123.

Santos, L. S., Souza, C. E., Monteiro, M, C., Prado, M. R. M. C., Prado Júnior, P. P., Ayres, F. A., \& Passos, C. M. (2019). Perfil social-profissional de enfermeiros e médicos da Atenção Primária à Saúde de uma microrregião geográfica. Revista Enfermagem Brasil, 18(4), 552-560.

Santos, R. S. S., Rosângela, A. P. F., Tabatha, T. F. M. S., \& Mauren, T. G. M. T. (2012). Atenção à saúde da criança: prática de enfermeiros da saúde da família. Revista Mineira de Enfermagem. 17(2), 331-339.

Silva, J. M. L., Monteiro, A. J. C., Coutinho, E. S., Cruz, L. B. S., Araújo, L. T., Dias, W. B., \& Costa, P. V. D. P. (2020). The instructional therapeutic toy as a tool in child cancer care. Research, Society and Development, 9(7), 1-14.

Silva, V. L., \& Pellenz, N. L. K. (2019). Os gestores de saúde na atenção primária a saúde versus capacitação para uma atuação satisfatória. Revista Científica Multidisciplinar Núcleo do Conhecimento, 4(9), 148-162.

Sobral, M. G. V., Pessoa, V. L. M. P., Florêncio, R. S., Solon, A. A. B., Bento, J. N. C., Cestari, V. R. F., \& Menezes, L. R. (2018). Elementos essenciais da consulta de enfermagem à criança e ao adolescente. Revista de Enfermagem UFPE, 12(12), 3464-3475.

Sousa, B. V. N., Lima, C. F. M., Félix, N. D. C., \& Souza F. O. (2020). Benefícios e limitações da sistematização da assistência de enfermagem na gestão em saúde. Journal of nursing Health, 10(2).

Souza, L. M., Silva, M. C. S., Zavalhia, S. R., Coppola. I. S., \& Rocha, B. P. (2018). Percepção de enfermeiros da Estratégia de Saúde da Família sobre segurança do paciente. Journal Nursing. Health, 8(2).

Sturmer, G., Pinto, M. E. B., Oliveira, M. M. C., Dahmer, A., Stein, A. T., \& Plentz, R. D. M. (2020). Perfil dos profissionais da atenção primária à saúde, vinculados ao curso de especialização em saúde da família una-sus no rio grande do sul. Revista Conhecimento Online, 12(1), 04-26.

Suto, C. S. S., Laura, T. A. O. F., \& Costa, L. E. L. (2014). Puericultura: a consulta de enfermagem em unidades básicas de saúde. Revista de Enfermagem UFPE, 8(9), 3127-3133.

Trindade, C. R., Santos, G. G., Mendonça, J. A., \& Rangel, S. D. (2019). Desafios do enfermeiro na consulta de puericultura para o estímulo do cuidado e autocuidado. Revista Científica Multidisciplinar Núcleo do Conhecimento, 07(01), 163-173.

Veiga, M. D. A. B., Sousa, M. C., \& Pereira, R. S. (2016). Enfermagem e o brinquedo terapêutico: vantagens do uso e dificuldades. Rev Eletrôn Atual Saúde, $3(3), 60-6$. 